\title{
Gene Set Enrichment Analysis (GSEA) of Toxoplasma gondii expression datasets links cell cycle progression and the bradyzoite developmental program
}

Matthew McKnight Croken', Weigang Qiu², Michael W White ${ }^{3}$ and Kami Kim ${ }^{1 *}$

\begin{abstract}
Background: Large amounts of microarray expression data have been generated for the Apicomplexan parasite Toxoplasma gondii in an effort to identify genes critical for virulence or developmental transitions. However, researchers' ability to analyze this data is limited by the large number of unannotated genes, including many that appear to be conserved hypothetical proteins restricted to Apicomplexa. Further, differential expression of individual genes is not always informative and often relies on investigators to draw big-picture inferences without the benefit of context. We hypothesized that customization of gene set enrichment analysis (GSEA) to T. gondii would enable us to rigorously test whether groups of genes serving a common biological function are co-regulated during the developmental transition to the latent bradyzoite form.
\end{abstract}

Results: Using publicly available T. gondii expression microarray data, we created Toxoplasma gene sets related to bradyzoite differentiation, oocyst sporulation, and the cell cycle. We supplemented these with lists of genes derived from community annotation efforts that identified contents of the parasite-specific organelles, rhoptries, micronemes, dense granules, and the apicoplast. Finally, we created gene sets based on metabolic pathways annotated in the KEGG database and Gene Ontology terms associated with gene annotations available at www.toxodb.org. These gene sets were used to perform GSEA analysis using two sets of published T. gondii expression data that characterized T. gondii stress response and differentiation to the latent bradyzoite form.

Conclusions: GSEA provides evidence that cell cycle regulation and bradyzoite differentiation are coupled. $\Delta g c n 5 A$ mutants unable to induce bradyzoite-associated genes in response to alkaline stress have different patterns of cell cycle and bradyzoite gene expression from stressed wild-type parasites. Extracellular tachyzoites resemble a transitional state that differs in gene expression from both replicating intracellular tachyzoites and in vitro bradyzoites by expressing genes that are enriched in bradyzoites as well as genes that are associated with the G1 phase of the cell cycle. The gene sets we have created are readily modified to reflect ongoing research and will aid researchers' ability to use a knowledge-based approach to data analysis facilitating the development of new insights into the intricate biology of Toxoplasma gondii.

Keywords: Gene expression, Transcriptome, Parasite, Bradyzoite, Tachyzoite, Differentiation, Development

\footnotetext{
* Correspondence: kami.kim@einstein.yu.edu

'Departments of Medicine, Microbiology \& Immunology and Pathology, Albert Einstein College of Medicine, 1300 Morris Park Avenue, 10461 Bronx, NY, USA

Full list of author information is available at the end of the article
}

\section{() Biomed Central}

(c) 2014 Croken et al.; licensee BioMed Central Ltd. This is an Open Access article distributed under the terms of the Creative Commons Attribution License (http://creativecommons.org/licenses/by/4.0), which permits unrestricted use, distribution, and reproduction in any medium, provided the original work is properly credited. The Creative Commons Public Domain Dedication waiver (http://creativecommons.org/publicdomain/zero/1.0/) applies to the data made available in this article, unless otherwise stated. 


\section{Background}

Toxoplasma gondii is an Apicomplexan parasite that is associated with encephalitis in the immunocompromised and chorioretinitis and birth defects in children exposed in utero. A central aspect of $T$. gondii virulence is its ability to persist as a latent slow-growing bradyzoite within tissue cysts. The reactivation of cysts, in the face of waning immune function, is a major cause of clinical toxoplasmosis. Despite the importance of this developmental transition the molecular mechanisms triggering differentiation are not understood. Expression analysis of bradyzoites and mutants unable to convert to bradyzoite have facilitated the identification of stage specific genes [1], but the critical signaling pathways have not yet been defined, in part because systems analysis tools are not available for this organism.

Gene expression analysis has revolutionized the analysis of biological problems, enabling an unbiased examination of gene expression on a genome-wide level. Initial analyses to detect biologically relevant but statistically robust changes in gene expression relied upon identification of changes in expression of single genes, usually using criteria that were designed to identify genes whose expression was altered most markedly and reproducibly. This resulted in lists of genes whose relation to each other was not obvious. As datasets expanded, methods to account for biological processes or genes whose expression were related in similar pathways or regulated by similar stimuli or perturbations were developed.

One of the most commonly used statistical methods is Gene Set Enrichment Analysis (GSEA) [2]. GSEA incorporates prior knowledge about biological states to create a priori gene sets that can be tested for concordant behavior in different biological conditions representing different phenotypes or different genotypes. Thus the co-regulation of genes that are functionally related, regulated by similar factors and conditions, or have another hypothesized biological link can be tested statistically.

The 8,814 genes of the $T$. gondii ME49 genome have been assigned Gene Ontology terms that assign gene products using a standard controlled vocabulary (http://www. geneontology.org) [3] that is meant to allow comparisons of gene attributes across species and databases. While GO terms are useful, many genes (48.6\%) have been annotated only as hypothetical proteins and a substantial number of genes belong to Apicomplexan-specific gene families, making GO vocabulary less useful for deducing the functions of many Apicomplexan genes. Most gene annotation of $T$. gondii has been computational with incorporation of community input via user comments. Extensive manually curated annotations like those available to model organism communities such as the Saccharomyces Genome Database available to the yeast community (http://www.yeastgenome.org/) [4] have not been uniformly incorporated into GenBank entries.

To develop gene sets that collate the extensive resources of ToxoDB (www.toxodb.org) [5], the primary community database, and published literature, we developed gene sets for our gene expression analysis, using the Molecular Signatures Database (MSigDB) (http://www.broadinstitute. org/gsea/msigdb/index.jsp) [2] that has been developed for use with GSEA, as a model. Application of these gene sets to analysis of preexisting datasets, illustrates a strong link between bradyzoite regulation and cell cycle and provides additional insight into the hierarchy of genes that lead to developmental transitions in T. gondii.

\section{Methods}

\section{Gene Set Enrichment Analysis (GSEA)}

Gene Set Enrichment Analysis is supported by the Broad Institute website (http://www.broadinstitute.org/gsea/index. jsp) [2] and includes versions compatible with Java, $R$ or Gene Pattern. All GSEA analyses presented here were performed using the Java GSEA implementation. No experiments involving animals, humans, or human material were performed and therefore no ethics approvals were required for this study.

\section{Development of gene sets}

The concept underlying GSEA is that genes that are somehow functionally linked will respond coordinately to a biological manipulation, in a manner that can be statistically detected and correlated to biological phenotype. These gene lists can be made using user-defined criteria. To define each new gene set, we first identified characteristics of interest in $T$. gondii and then identified genes that have or are associated with this characteristic. The default parameters of GSEA using gene lists of 15 to 500 genes were used as our target size in generating the new gene sets. KEGG pathway genes for $T$. gondii were downloaded from the KEGG site (http://www.genome.jp/kegg-bin/show_organism?menu_type=pathway_ma ps\&org=tgo) [6]. GO terms and genes were downloaded from www.toxodb.org. Additional information about $T$. gondii genes and gene lists were provided by Jeroen Saeij (MIT) who had independently culled additional gene set information from the proteomics and gene expression literature as well www.toxodb.org. In the case of KEGG pathways, the gene sets are primarily metabolic pathways although KEGG also has an annotated list named "Toxoplasmosis" that consists of genes from $T$. gondii linked to pathogenesis or host interaction, as well as genes for host pathways affected. Similarly, gene sets derived from GO terms are related either by genes' identified biological process, cellular component, or molecular function. 
Manually curated gene lists provided by the Toxoplasma research community and incorporated in into the community database www.toxodb.org were obtained from Omar Harb, University of Pennsylvania, and compared with lists generated by text searches of Toxodb using key words apicoplast, rhoptry, microneme, dense granule, ROP, RON, MIC, GRA $[5,7,8]$. The gene list obtained from www. ToxoDB.org using "apicoplast" as a key word was very large and it was unclear whether all were genuine apicoplast proteins, so a community annotation gene list was used instead. Organellar gene sets were organized by subcellular localization rather than biological processes (i.e. where they are, rather than what they are doing).

To generate gene sets associated with particular stages of the parasite life cycle, we examined gene expression data and grouped co-regulated genes into relevant gene sets. Two previous studies used microarray experiments to identify genes associated with bradyzoite [9] and sporozoite [10] developmental stages of the life cycle were used to develop "tachyzoite", "bradyzoite" and "sporozoite/oocyst" gene lists. The strain used for these studies, the Type II M4 strain, was maintained in continuous cat to mouse to cat to maintain competence for all life cycle transitions, but as a consequence, in vitro populations maintained a background level of spontaneous differentiation and were not completely pure "tachyzoites" or "bradyzoites". Type II strains are most frequently used to model the biology of the bradyzoite differentiation process. Duplicate biological replicates of mRNA were used by the authors to probe microarrays, and our initial gene sets relied on the authors' statistical inferences. Genes that were significantly up-regulated under the same conditions were placed together into gene sets, while those that were down-regulated were placed into an opposing gene set (e.g. tachyzoites and bradyzoites). It should be noted that since the gene sets are user defined, the gene sets used for GSEA can be overlapping based upon user-defined criteria. To view the genes that overlap in our gene sets, a complete list of genes is provided in Additional file 1 as a spread sheet (Table S1 GeneMembership). Gene lists used for this analysis (Version 6 gene IDs) and for the current $T$. gondii genome release (Version 10) are also provided in Additional files 2 and 3.

\section{Test data}

To test the usefulness of our newly developed $T$. gondii gene sets, we used published microarray data sets to identify pathway enrichments associated with the development from tachyzoites to bradyzoites in T. gondii. The user guide for GSEA recommends 7 replicates, which are generally not available, so we used datasets with at least 3-4 biological replicates. These included the data set GSE23174 [11], which compared RH (type I) in vitro bradyzoites to in vitro intracellular tachyzoites as well as extracellular tachyzoites. These $\mathrm{RH}$ strain parasites lack the UPRT gene and differentiate more readily to in vitro bradyzoites than their wild-type parent. Bradyzoite induction was induced by low $\mathrm{CO}_{2}$, high $\mathrm{pH}$ culture conditions (alkaline stress) [11].

We also tested data set GSE22100 for enrichment of our gene sets [12], which was used to support a study of GCN5A function. The authors characterized the transcriptome of a RH strain mutant lacking GCN5A ( $\triangle g c n 5 A)$, one of two $T$. gondii GCN5 histone lysine acetyltransferases [12]. The authors showed that, while the wild-type parasites and $\triangle g c n 5 A$ mutants show virtually identical transcriptomes when grown in tachyzoite conditions, when grown in alkaline stress wild-type parasites up-regulate several key markers of bradyzoite differentiation while the $\triangle g c n 5 A$ mutants fail to upregulate bradyzoite markers [12].

Expression data for both sets of experiments was extracted and normalized from CEL files using the ExpressionFileCreator module from Gene Pattern a software platform available at the website of the Broad Institute (http://www.broadinstitute.org/cancer/software/genepattern/) [13]. We used RMA normalization.

\section{Results and discussion \\ Cell cycle}

The cell cycle of Toxoplasma gondii is known to be divergent from other model eukaryotes. It has a greatly abbreviated or absent $G_{2}$ phase with DNA synthesis (S-phase) coupled directly to mitosis (M phase) [14]. Behnke and colleagues identified two sub-transcriptomes, $G_{1}$ and $S / M$, based on a microarray study of synchronized parasites, sampling mRNA levels every hour for twelve hours [14]. The replication time of the RH strain of $T$. gondii is reported to be about eight hours [15]. Based on the microarray results from the twelve, one-hour time points, the authors fit a spline model to estimate transcript levels throughout the cell cycle in higher resolution [14]. From the twelve actual time points, the spline model extrapolates sixty "splined" time points, each representing a twelve minute increment. Using this spline model, we identified peak times of expression for cell cycle regulated genes.

For each gene, we defined peak times of expression as greater than the mean expression across all splined time points plus 1.25 standard deviations. Genes assigned to each splined time point become our gene sets. Using these criteria, only 1,927 genes of the 2,833 cell cycle regulated genes are included in the gene sets. These criteria allowed us to generate gene sets for GSEA within the recommended size limits, between 15 and 500 genes. Figure 1A illustrates sizes of the generated gene sets for $\mathrm{G}_{1}$ and $\mathrm{S} / \mathrm{M}$ and illustrates the previously reported major peaks of cell cycle gene expression [14]. A list of all genes and their membership in each GSEA gene set 


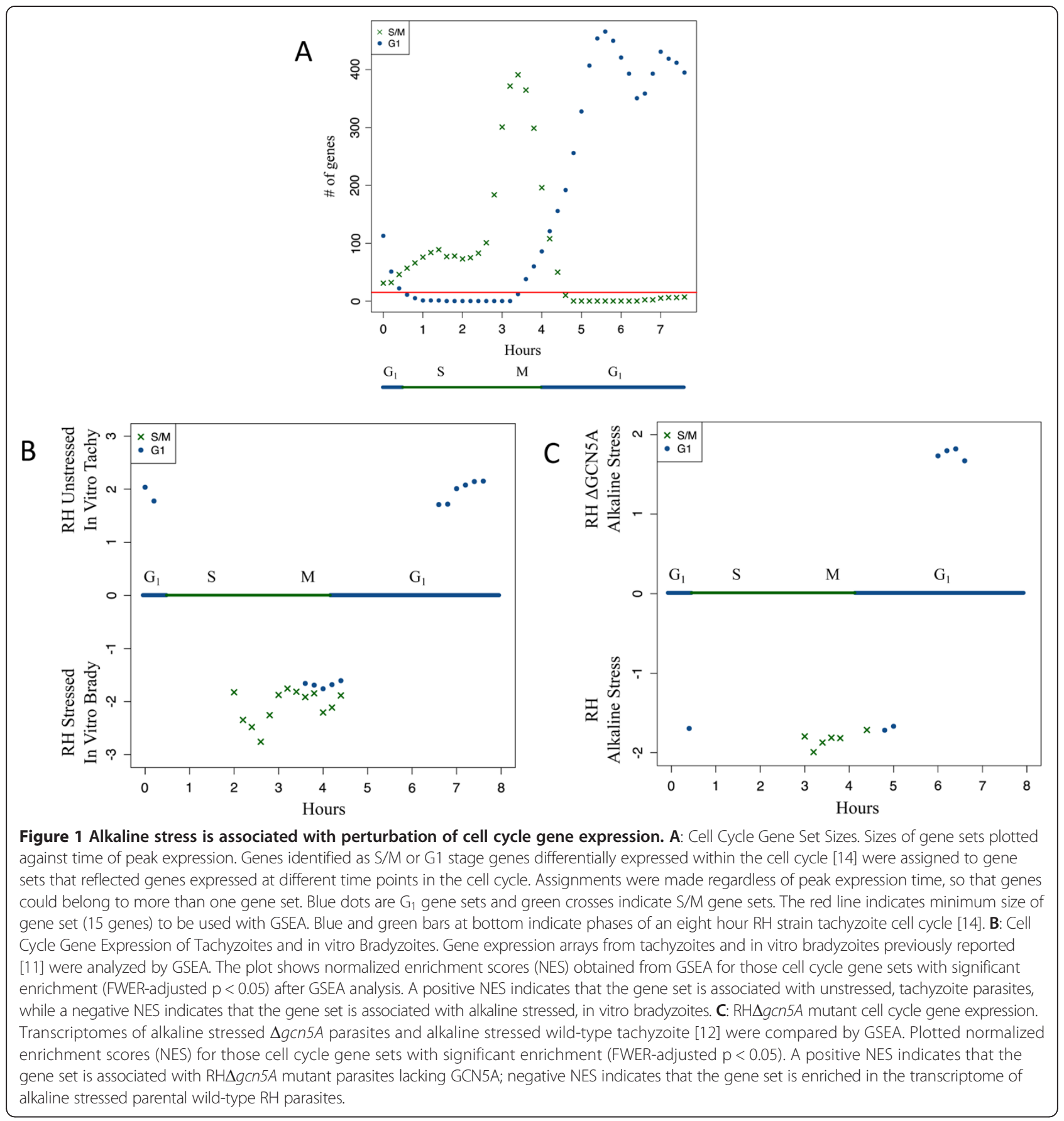

is in Additional file 1 and full gene sets for each cell cycle time point are available in Additional files 2 and 3 as gmt files compatible with GSEA.

We ran GSEA using the cell cycle gene sets against two different published microarray experiments. These datasets were chosen because the experiments were performed in different laboratories but used RNA extracted from the common laboratory strain $\mathrm{RH}$ and expression profiling was performed on the community generated Toxoplasma chip on the Affymetrix microarray platform [16]. These datasets illustrate cases where parasites modulate steadystate mRNA levels in response to environmental changes and test how genetically modified parasites respond to the same stimulus. We reasoned that our gene sets in combination with GSEA might provide additional insights into how transcriptional differences reflect biological responses to an environmental stress that is the standard laboratory manipulation for induction of in vitro bradyzoites.

In the first expression experiment reported by Lescault

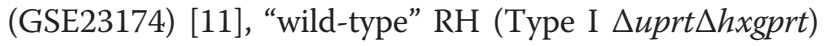


parasites were grown in tissue culture under $\mathrm{pH}$ neutral, tachyzoite-conducive conditions $\left(5 \% \mathrm{CO}_{2}\right)$ or under alkaline, bradyzoite-inducing conditions (low $\mathrm{CO}_{2}$ ) [11]. $\mathrm{RH}$ strain parasites lacking UPRT are more sensitive to alkaline stress induction of bradyzoites [17] whereas most laboratory strains of $\mathrm{RH}$ do not complete bradyzoite differentiation, although they can express classic bradyzoite markers such as BAG1 when grown under alkaline stress conditions for 3-4 days. Comparing intracellular tachyzoites to in vitro "bradyzoites," gene sets associated with the S/M phase, are strongly up-regulated under alkaline stress "bradyzoite" conditions. These observations are consistent with the prolongation of the $S / M$ phase associated with bradyzoite differentiation noted previously [18]. This prolonged S/M is distinguished by the coexpression of both tachyzoite (SAG1) and bradyzoite (BAG1) by the same parasite [18]. Asynchronous tachyzoite cultures are usually typically predominantly (about $70 \%)$ in G1 of the cell cycle. Notably, some gene sets classified as $G_{1}$ whose temporal expression coincides with the $\mathrm{S} / \mathrm{M}$ phase are up-regulated in the in vitro bradyzoites (Figure 1B).

We also tested for perturbation of cell cycle associated genes using data set GSE22100 that compared the transcriptomes of mutant parasites lacking GCN5A, a histone acetyltransferase, to wild type parasites (in this case RH $\Delta$ hxgprt) grown in alkaline stress [12]. GCN5A is required for activation of bradyzoite-specific genes and $\triangle g c n 5 A$ mutant parasites fail to express bradyzoite differentiation markers in alkaline culture and exhibit transcriptomes that are "tachyzoite-like" [12]. Using GSEA, we observe a significant enrichment of S/M gene sets within the wild-type, alkaline stressed parasites and G1 gene sets by the $\Delta g c n 5 A$ mutant parasites exposed to alkaline stress (Figure $1 \mathrm{C}$ ), indicating that under alkaline stress, the expression of $\triangle g c n 5 A$ mutant cell-cycle regulated genes is more similar to tachyzoites. This may indicate that one of the mechanisms by which GCN5A regulates the stress response is via regulation of cell cycle checkpoints.

\section{Oocyst maturation}

Following the sexual phase of the Toxoplasma life cycle, oocysts are released in the cat's feces [10]. Sporulation of fecal oocysts leads to infective, environmentally hardy parasites that can contaminate human food and water supplies. These oocysts are highly infectious and, when ingested, can cause clinically symptomatic infection, usually chorioretinitis, in both immunocompetent and immunosuppressed individuals. Using expression microarrays, Fritz et al. profiled a type II (strain M4) oocyst transcriptome immediately and at days four and ten after oocyst release [10]. By microscopy, day zero oocysts are immature and completely unsporulated. Day four oocysts begin to develop a more mature structure, but less than half have sporozoites. Day ten oocyst are considered mature, in that almost all have two well developed sporocysts and the "vast majority" had discernible sporozoites [10]. Following the development of the oocyst, a serial transcriptomic study was performed to identify several different patterns of gene expression during oocyst development.

We assigned genes to thirteen sporozoite gene sets based on changes in expression between time points as described in (Additional file 4: Figure S1). Five of these are "core" oocyst gene sets, featuring genes whose expression peaked during one or two of the observed time points (Figure 2A). These gene sets were called "early-middle", "middle", "middle-late", "late", and "early-late" oocyst genes. Too many genes fell into the "early" gene set to be useful for GSEA. An additional seven gene sets were created to encompass genes with more complex patterns of expression. These gene sets are provided as extended oocyst gene sets in Additional files 1, 2 and 3 and their corresponding patterns of expression are illustrated in Additional file 4.

We ran GSEA with the previously described microarray experiments (GSE23174 and GSE22100) against the oocyst gene sets. Surprisingly, in the comparison of the Lescault "tachyzoites" with "bradyzoites" [11], genes associated with the middle-late stage of sporulation were enriched in the in vitro bradyzoite parasite population (Figure 3B). In contrast, GSEA analysis of the $\triangle g c n 5 A$ mutant parasites showed no significant enrichment for any oocyst gene set (data not shown). Too little is known about the sporozoite developmental program to definitively interpret the biological significance of these results, but one can hypothesize overlap between the in vitro bradyzoite and oocyst transcriptomes or that common genes are induced during any developmental transition. The $\Delta g c n 5 A \mathrm{RH}$ background strain (RH $\Delta$ hxgprt) expresses stress markers associated with the bradyzoite transition, but does not complete differentiation to bradyzoites as defined by presence of the cyst wall [12].

\section{Bradyzoite differentiation}

The ability of Toxoplasma gondii to differentiate into bradyzoites and persist within quiescent tissue cysts is a key survival strategy. The latent bradyzoite form allows the parasite to evade the host immune response while awaiting contact with a new host. Recrudescence of encysted parasites is responsible for most clinical disease and can cause lethal encephalitis in immunocompromised individuals. Therefore, the molecular mechanisms responsible for differentiation between tachyzoites and bradyzoites are of keen interest.

Prior work has suggested that bradyzoite differentiation and cell cycle are coupled, with the first detectable initiation of the differentiation program occurring in S/M phase, just prior to mitosis. As bradyzoites mature, their 


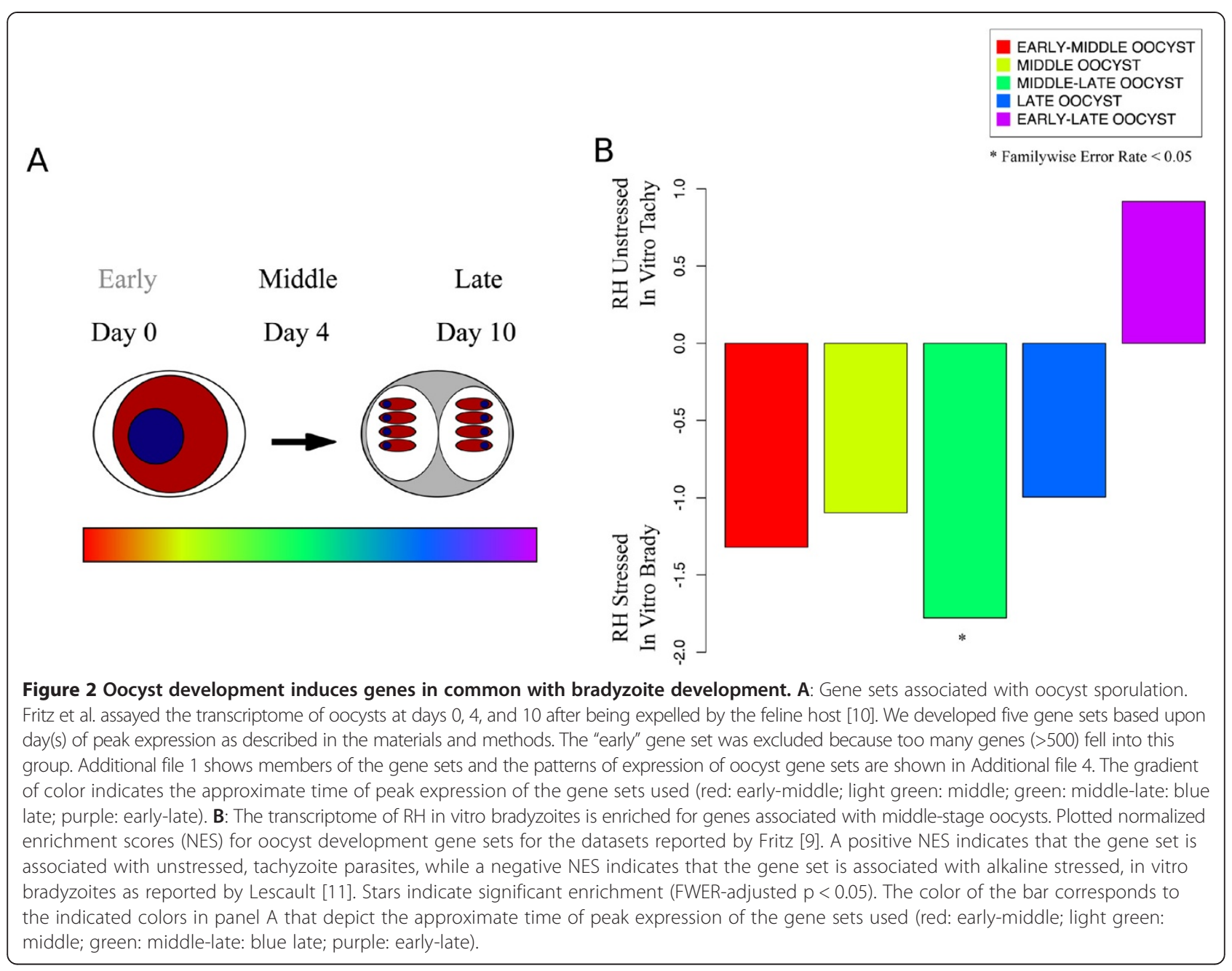

metabolism slows. Parasites induced to differentiate in vitro tend to have a slowing of the S/M phase and parasites with dual expression of the bradyzoite marker BAG1 and the tachyzoite marker SAG1 are more likely to be in S/M phase [18]. Induction of bradyzoite markers is observed when tachyzoites are incubated as extracellular tachyzoites for prolonged periods [19], suggesting that these parasites are able to sense alterations in their environment and induce bradyzoite gene expression. Based upon the cell cycle profile of extracellular parasites, this signal is likely to be sensed by parasites in the G1 phase of the cell cycle. Bradyzoite differentiation requires replication [1], but eventually, mature bradyzoites within in vivo tissue cysts complete mitosis and then exit the cell cycle, entering the $\mathrm{G}_{0}$ phase [18].

In addition to greatly slowed metabolism and replication, the other major feature of bradyzoites is cyst formation. During encystation within the host cell, the parasitophorous vacuole is lined with a glycosylated cyst wall, and the parasite expresses a stage-specific antigens, notably BAG1 (bradyzoite antigen 1) and CST1, a glycoprotein present in the bradyzoite cyst wall [20,21] Detection of these markers is considered diagnostic of bradyzoite differentiation. T. gondii also expresses paralogous metabolic genes associated with a particular stage of life. For instance, enolase 2 and lactose dehydrogenase 1 are expressed during tachyzoite development while enolase 1 and lactose dehydrogenase 2 are up-regulated following differentiation [1].

To obtain a more comprehensive view of bradyzoite differentiation, Buchholz and colleagues assayed the transcriptomes of type II (strain M4) bradyzoites [9]. They examined in vitro bradyzoites at four and eight days post induction, in vivo bradyzoites harvested from mouse brains twenty-one days post-infection, and compared the transcriptome of each bradyzoite type to the transcriptome of tissue culture grown tachyzoites [9]. This study catalogued changes in mRNA expression between tachyzoites and bradyzoites, differences between in vitro and in vivo derived bradyzoites, as well as temporal changes in the parasite transcriptome during bradyzoite development in tissue culture [9]. 


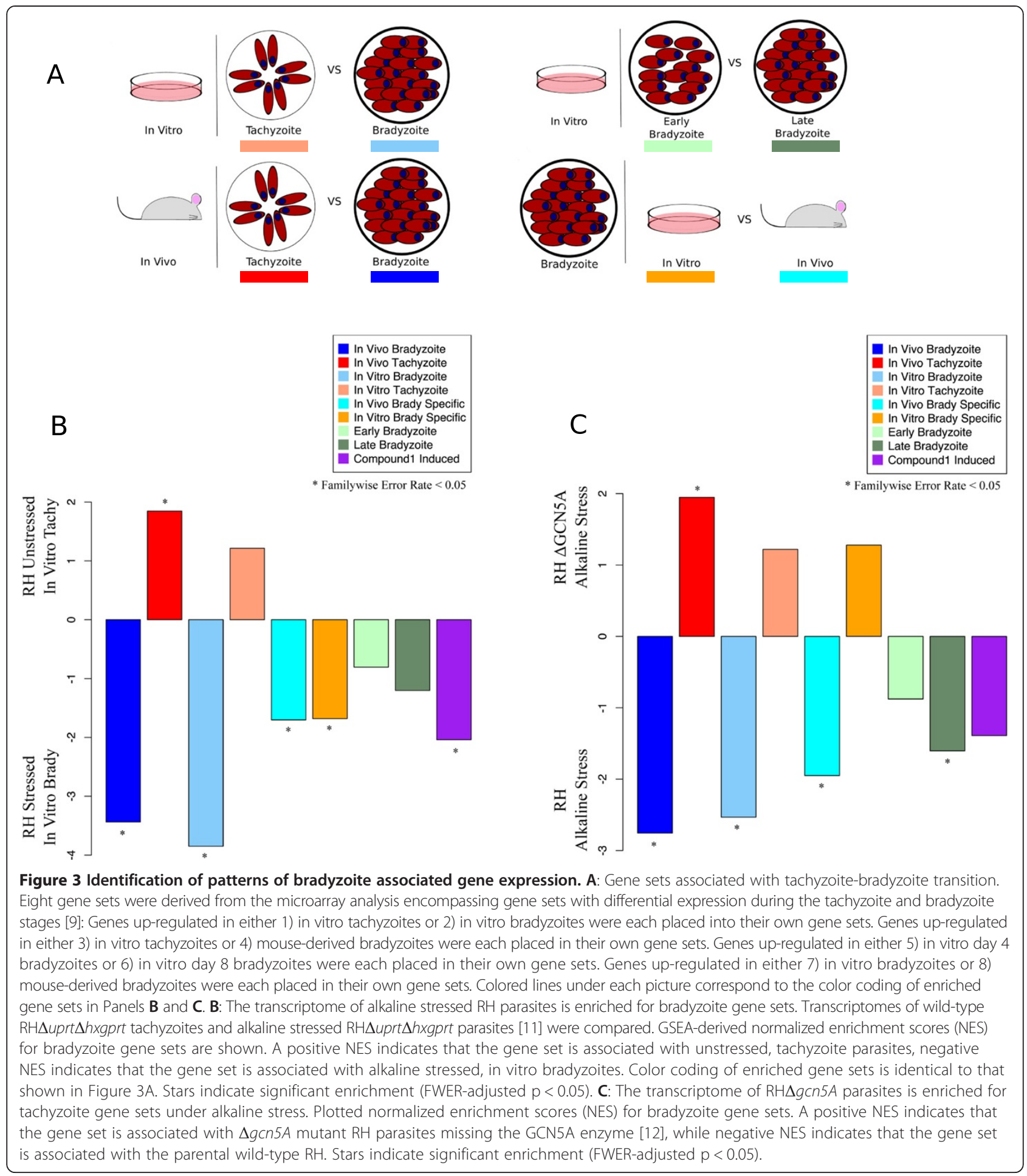

We created eight bradyzoite gene sets based on four different pair-wise comparisons illustrated in Figure 3A. These gene sets were designed to test whether bradyzoite differentiation had occurred, to identify which if genes associated with early or late stages of bradyzoite differentiation were expressed, as well as whether we could detect differences in expression of genes differentially expressed between mouse-derived cysts and alkaline/ low $\mathrm{CO}_{2}$ in vitro cysts. As a final bradyzoite gene set, we used the set of genes enriched by treatment of parasite cultures with Compound 1, a kinase inhibitor that induces bradyzoite formation in Type II and Type III strains [22]. 
The Compound 1 gene set is based on previously published experiments examining differentiation in three different strains. Significance calls were made based on ANOVA analysis of all three strains [22], rather than pairwise comparisons like the other eight gene sets. We identified many common markers of bradyzoite differentiation within our bradyzoite gene sets. The complete gene sets can be found in Additional files 1, 2 and 3.

GSEA was able to clearly distinguish between tachyzoite and bradyzoite populations as characterized by Lescault (GSE23174) [11], but could not successfully identify these as in vitro bradyzoites and tachyzoites (Figure 3B). Further, the in vitro bradyzoite population was enriched for both in vitro and in vivo specific bradyzoite markers. This suggests that, although these genes are differentially expressed by in vitro and in vivo bradyzoites, both sets of genes are more highly expressed in bradyzoites than tachyzoites, regardless of the origin of the bradyzoite. The Compound 1 induced gene set was significantly enriched in the bradyzoite population.

GSEA classified the transcriptome of $\triangle g c n 5 A$ parasites as tachyzoite-like and that of the parental $\mathrm{RH}$ strain as bradyzoite-like (Figure 3C). This is consistent with Naguleswaran and colleague's observation that the mutant parasites fail to tolerate alkaline stress conditions [12]. Curiously, wild-type $\mathrm{RH}$ is enriched for in vivo specific bradyzoite markers, but not in vitro specific genes, suggesting subtle differences in mRNA expression profiles. It also interesting to note that in vitro specific bradyzoite gene set is enriched in the more tachyzoite-like $\Delta g c n 5 A$ parasites, albeit non-significantly $(\mathrm{p}=0.259)$. The basis of this enrichment is unclear. The genes most up-regulated are diverse, but include DNA replication factors, protein translational machinery, and other apparently cell-cycle regulated genes.

\section{Subcellular localization}

Prior studies in both Plasmodium [23,24] and T. gondii [14] showed that steady state mRNA levels are present "just in time" with related metabolic genes or organellar genes frequently expressed at similar points in the cell cycle. We collected genes associated with the secretory organelles as prior microarray analysis of the gene products that localize to: rhoptries, micronemes, and dense granules were often coexpressed. In addition, we used a set of genes that was a part of the community annotation of gene products that localize to the apicoplast (obtained from Omar Harb, www.toxodb.org). Taken together, we have gene sets describing many of the cellular structures specific to Apicomplexans. These gene lists can be revised as experimental evidence accumulates about these organelles, and additional hypothetical genes are assigned to secretory organelles, the apicoplast, or the mitochondrion [25].
The in vitro bradyzoites of Lescault and colleagues (GSE23174) [11] show enrichment for gene products localized to micronemes and rhoptries, while their tachyzoite counterparts show up-regulation of denizens of the apicoplast (Additional file 5: Figure S2A). We did not observe any significant enrichment in either the wildtype or $\Delta g c n 5 A$ alkaline stressed parasite populations for any of the examined organellar gene sets although the trends of gene expression enrichment were evident (Additional file 5: Figure S2B).

\section{Metabolic pathways}

Both the Gene Ontology (GO) and Kyoto Encyclopedia of Genes and Genomes (KEGG) projects have been applied to the Toxoplasma genome to better characterize the functions of and the relationships between genes. KEGG contains a database of metabolic pathways to which homologous genes from any species may be mapped onto a "canonical" pathway [6]. Although there are obvious drawbacks to this approach when dealing with a divergent eukaryote like $T$. gondii, it does provide a framework for examining parasite metabolism. Of the 87 annotated KEGG pathways present in Toxoplasma, only 33 have an appropriate size for GSEA (See Additional files 1, 2 and 3). Further manual annotation of metabolic pathways and comparison with other databases such as the LAMP database [26], will likely improve our knowledge of Toxoplasma metabolism. It should be noted that mis-assignment of one or two genes within these pathways would not necessarily affect the statistical ability of GSEA to detect co-regulation, if the majority of genes are correctly assigned and their steady state mRNA levels are co-regulated. GO terms are an effort to standardize descriptions of gene functions and place these functions into hierarchies, called ontologies [3]. This type of organization readily lends itself to GSEA. Of 325 GO terms assigned in Toxoplasma, 215 have an appropriate size for enrichment analysis (Additional file 1 ). For analysis of the datasets in this manuscript, GSEA using KEGG gene lists were more informative, but GO analysis is commonly used for inference of gene pathways in microarray analysis.

The tachyzoites tested by Lescault [11] (GSE23174) show significant enrichment of nine different KEGG pathways, while in vitro bradyzoites had enrichment of only one gene set (Figure 4A). This follows, since tachyzoites are highly metabolically active while bradyzoites are comparatively quiescent. These data may indicate key pathways that are affected during the transition to bradyzoites. We also see enrichment of transcripts associated with ATP production via oxidative phosphorylation and the citric acid cycle in tachyzoite parasites as well as transcription and translation machinery. Interfering with these pathways via drug, inducible mutation, or substrate 


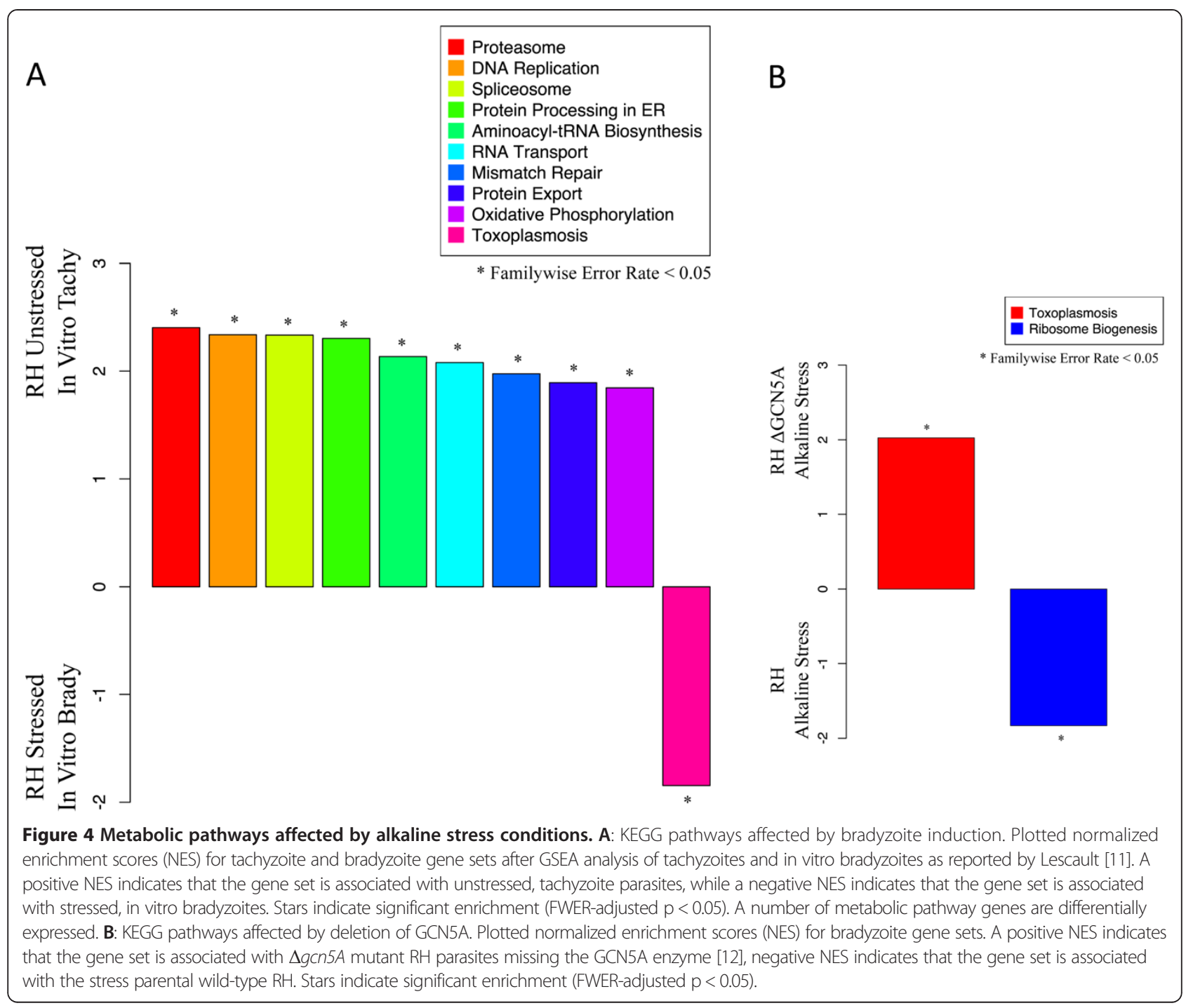

starvation could induce bradyzoite differentiation. The generation of the atovoquone-resistant mutant R5, which differentiated more readily to bradyzoites, provides support for this hypothesis [27].

Similar experiments or reanalysis of previous experiments should provide new information about the particular pathways and molecular decision making that leads to life cycle development within Toxoplasma. It is also likely that important bradyzoite-specific metabolic pathways are not annotated, and manual curation of gene sets may facilitate further insights into the regulation of stagespecific metabolic pathways during different life cycle stages.

When alkaline stressed, the tachyzoite-like $\Delta g c n 5 A$ mutants (GSE22100) and their differentiated wild-type counterparts [12] were enriched for only one gene set derived from KEGG terms (Figure 4B). The only KEGG gene set enriched in stressed $\triangle g c n 5 A$ mutants was a pathway titled "Toxoplasmosis". This pathway consists largely of cell surface adhesins (some specific to bradyzoites, others peculiar to tachyzoites) as well as many secreted factors that interfere with host cell signaling. The pathway also contains many host cell signaling factors, mainly related to the immune response. Since the "Toxoplasmosis" gene set includes several different classes of genes, its use with GSEA is not entirely appropriate. In this case, enrichment is based almost entirely on the up-regulation of stage-specific SRS domain-containing proteins.

In the alkaline stressed wild-type parasites, "Ribosome Biogenesis" genes were up-regulated in comparison to unstressed parasites. The differences in induction of metabolic pathway genes in these two datasets in response to alkaline stress may reflect the differences between the two background strains in stress response including possibly the induction of stress-induced translational control that has been implicated in bradyzoite cyst formation [28,29]. 


\section{Extracellular tachyzoites}

Based on their mRNA expression experiments (GSE23174), Lescault and co-authors identified extracellular tachyzoites as a distinct stage of Toxoplasma asexual reproduction [11]. Using our newly developed gene sets, we further characterized the differences between extracellular tachyzoites, intracellular tachyzoites, and in vitro bradyzoites.

When extracellular tachyzoites are compared to intracellular tachyzoites, bradyzoite gene sets, derived from in vitro and in vivo bradyzoite gene sets, are enriched in extracellular tachyzoites (Figure 5A). The in vivo tachyzoite gene set, but not the in vitro tachyzoite gene set, is enriched in the transcriptome of intracellular tachyzoites compared to extracellular parasites. However, when the transcriptome of extracellular tachyzoites is compared to the transcriptome of in vitro bradyzoites, bradyzoite gene sets are significantly enriched in the transcriptome of the bradyzoites, while tachyzoite gene sets are enriched in neither transcriptome (Figure 5B).

The transcriptome of extracellular tachyzoites also exhibit an apparent enrichment of $G_{1}$ genes consistent with a post-mitotic cell cycle arrest or delay, when compared to both intracellular tachyzoites and intracellular in vitro bradyzoites. The timing of this arrest is consistent with cell-cycle exit in the $G_{0}$ phase. Flow cytometry comparing DNA content of extracellular and intracellular tachyzoites shows a much greater proportion of haploid parasites $\left(G_{1}\right)$ in the extracellular group (Additional file 6: Figure S3). This confirms that extracellular parasites exist almost exclusively outside of the $\mathrm{S}$ phase and that their transcriptome is different from that of proliferating intracellular tachyzoites [30].

Taken together, these GSEA results support the hypothesis that extracellular tachyzoites represent a distinct state of parasite asexual development [11]. The GSEA results further suggest a continuum of stress response or stress preparedness between the three developmental stages examined in these experiments. At one end of the spectrum is the bradyzoite stage, which provides long-term defense against stress and host immunity, with replicating intracellular tachyzoites at the other end. The transcriptome of the extracellular tachyzoite stage is in the middle of this spectrum, reflecting the moderate stress encountered when the parasite is navigating the extracellular milieu to find a new host cell. Unlike the intracellular tachyzoite, these parasites lack the protection of a parasitophorous vacuole and host cell. However, unlike the bradyzoite stage, the extracellular stage is typically only short-term. Notably, sporozoites and bradyzoites, the other invasive zoite forms that must find new host cells also predominantly have $G_{1}$ DNA content, a state that is likely to be the most advantageous for the parasite to successfully invade a new host cell without committing to proliferation. Cell cycle arrest may also be accompanied by upregulation of genes that enable the parasite to survive environmental stress prior to finding and invading a suitable host, with down-regulation of genes primarily required during intracellular replication.

\section{Conclusions}

Deciphering the large amount of Toxoplasma transcriptomic data currently available requires integrative approaches to data analysis that incorporate experimental data and bioinformatics analysis. By establishing $T$. gondii gene sets, our aim is to create a framework to view changes in biological processes, rather than just individual genes, and drive hypothesis generation.

GSEA is a powerful tool for such knowledge-based analysis techniques and a considerable amount of relevant knowledge exists for $T$. gondii, but leveraging experimental data into gene sets can be a difficult task. The gene sets collected here describe a number of biological processes potentially linked to parasite virulence or disease pathogenesis. Because of our long-standing interest in the relationship of bradyzoite differentiation and cell cycle, we began have focused on biological aspects of these processes, particularly those specific to the parasite. Apicomplexanspecific or unique T. gondii genes may be associated with virulence or parasite metabolism, but differences in their expression are will be difficult to translate from gene sets developed for other model organisms. While KEGG and GO provide useful classifications for functionally related genes, assignment to KEGG pathways or GO terms are dependent on sequence homology between T. gondii and other eukaryotes. Given the evolutionary divergence of the Apicomplexa and the incomplete annotation of parasite genomes, new integrative approaches are needed that incorporate multiple lines and types of experimental data.

While these initial efforts have relied heavily on microarray expression data to develop $T$. gondii gene sets, expression analysis is by no means the only means for discovering functional links between genes. Protein-protein networks, inferred computationally or derived from experimental approaches such as proteomics analysis of immunoprecipitated complexes or yeast two-hybrid screens, may prove to be useful gene sets. A growing wealth of ChIP-chip and ChIP-seq data makes it possible to identify genes related by common epigenetic mark or bound by the same transcription factor. Finally, integration of knowledge from experiments validating existing gene sets can generate new groupings in an iterative manner. A major advantage of all of these strategies is that gene sets are user defined and therefore can evolve and be tested experimentally for statistical robustness.

The Molecular Signatures Database (MSigDB) curates many human gene sets for use with GSEA. MSigDB organizes the gene sets into seven separate collections. Many of these collections relate to oncology and immunology or 


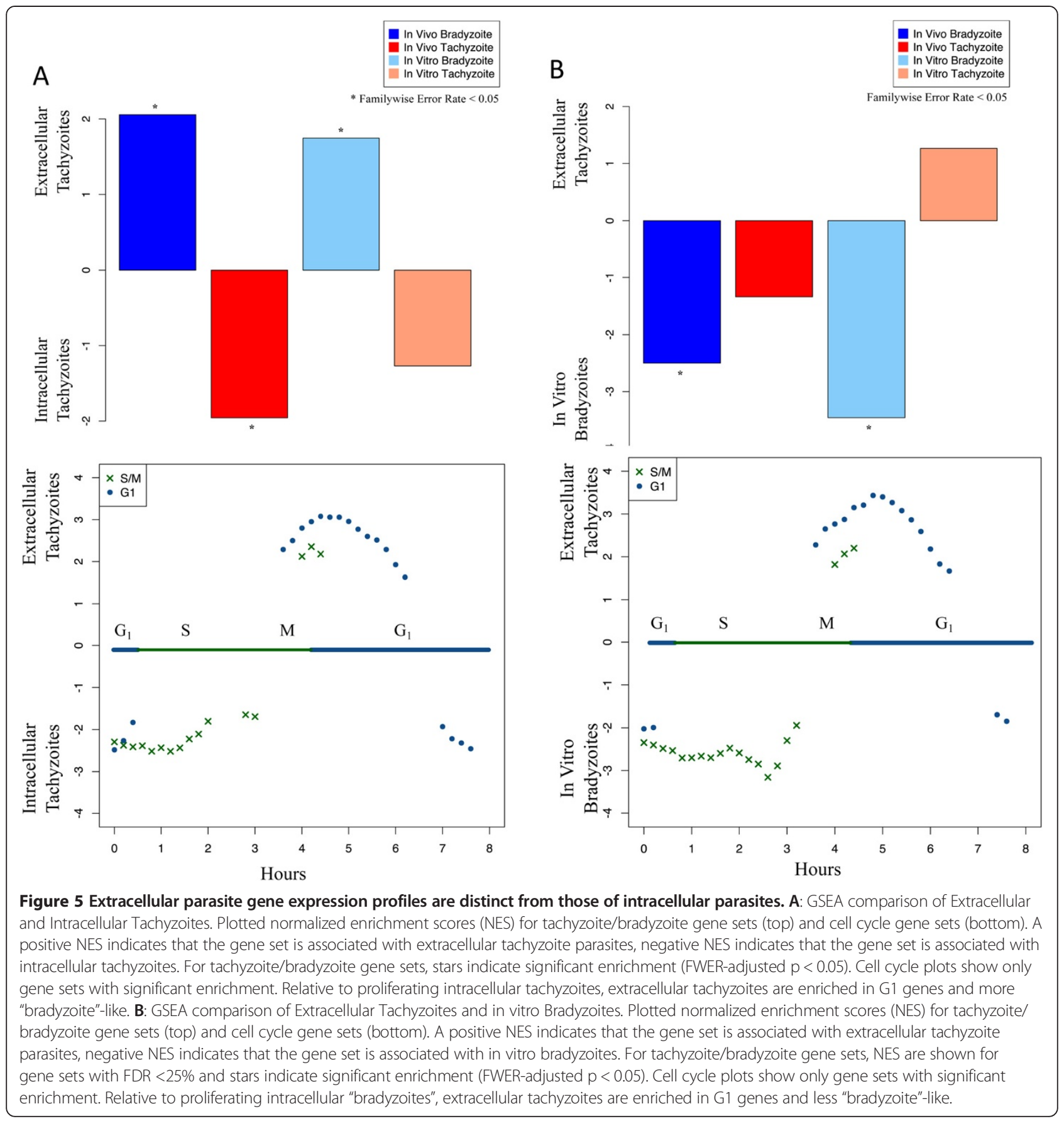

other topics specific to metazoa that are not applicable to Toxoplasma, but others model gene sets that would be useful for Toxoplasma. In particular, genes that possess the same cis-regulatory elements may be grouped together as potential targets of the same transcription factor. Identification of cis-regulatory elements is ongoing within Apicomplexan parasites. A large family of candidate transcription factors, the ApiAP2, are conserved throughout the phylum Apicomplexa [31,32]. The DNA binding specificities for most ApiAP2 in Plasmodium falciparum have already been described [33] and a similar effort for the $T$. gondii ApiAP2 binding specificities is nearly complete (Ranjan et al., in preparation). Simultaneous motif analysis and mapping of binding motifs onto the genome, should enable reconstruction of transcriptional regulatory pathways and produce sets of genes regulated by the same identical transcription factor. The gene sets described here represent a powerful new set of tools for deep analysis of Toxoplasma expression data with the GSEA software. 


\section{Additional files}

Additional file 1: Table S1. GeneMembership. Gene sets used for GSEA. All T. gondii genes with their Version 6 ID are listed. The first column indicates classic tachyzoite (shaded blue) and bradyzoite markers (shaded yellow). Columns indicate the name of the gene set.

\section{Additional file 2: GMT files formatted for use in GSEA (Version 10).} Genome details were downloaded from the $T$. gondii genome resource www.toxodb.org. The current genome Version 10 (R10) formatted Gene ID's are provided. Detailed instructions for implementation of GSEA are available at the Broad Institute website (http:/www.broadinstitute.org/gsea/index.jsp).

Additional file 3: GMT files formatted for use in GSEA (Version 6). Genome details were downloaded from the $T$. gondii genome resource www.toxodb.org. Genome Version 6 (R6; used for this manuscript) formatted Gene ID's are provided. Detailed instructions for implementation of GSEA are available at the Broad Institute website (http://www.

broadinstitute.org/gsea/index.jsp).

Additional file 4: Figure S1. Oocyst gene sets. Patterns of expression used to categorize core oocyst gene sets and extended oocyst gene sets. Expression data are from Fritz et al. [10] who profiled a type II (strain M4) oocyst transcriptome immediately and at days four and ten after oocyst release.

Additional file 5: Figure S2. Expression of organellar gene sets. Figure S2A: Bradyzoite induction leads to differential expression of organellar gene sets. Plotted normalized enrichment scores (NES) for bradyzoite gene sets from Lescault et al. [11]. A positive NES indicates that the gene set is associated with unstressed, tachyzoite parasites, while a negative NES indicates that the gene set is associated with alkaline-stressed in vitro bradyzoites. Stars indicate significant enrichment (FWER-adjusted $p<0.05$ ). Figure S2B Alkaline-stressed RH $\Delta g c n 5 A$ parasites are not enriched for any subcellular gene sets. Plotted normalized enrichment scores (NES) for organellar gene sets. A positive NES indicates that the gene set is associated with alkaline-stressed $\mathrm{RH} \Delta g \mathrm{~g} n 5 \mathrm{~A}$ parasites [12], while a negative NES indicates that the gene set is associated with the alkaline-stressed parental wild-type $\mathrm{RH}$. None of the organellar gene sets tested had statistically significant enrichment (FWER-adjusted $p<0.05$ ).

Additional file 6: Figure S3. Cell Cycle Profile (DNA Content) of Tachyzoites. Extracellular lysed tachyzoites or intracellular tachyzoites harvested from human foreskin fibroblasts were fixed and labeled with propidium iodide and analyzed by flow cytometry. The extracellular parasites are enriched for $1 \mathrm{~N}$ DNA content consistent with predominant $G_{1}$ or $G_{0}$ state. $S$ phase parasites have intermediate amounts of DNA, whereas $G_{2}$ or M parasites will have close to $2 \mathrm{~N}$ DNA content. Intracellular parasites are asynchronously proliferating with parasites in each of the major cell cycle stages, but are predominantly in G1.

\section{Competing interests}

The authors declare no competing interests.

\section{Authors' contributions}

MMC conceived the study, performed all data analysis, and wrote the manuscript. WQ performed initial GSEA feasibility studies. MWW provided cell cycle datasets, intellectual input, and edited the manuscript. KK conceived the study, supervised MMC, and wrote the manuscript. All authors read and approved the final manuscript.

\section{Acknowledgements}

This research was supported by National Institutes of Health grants R01Al087625 (KK), RC4Al092801 (KK, MWW), R01Al077662 (MWW) and R01Al089885 (MWW). MMC was supported by the Training Program in Cellular and Molecular Biology and Genetics, funded by NIH T32 GM007491 awarded to the Albert Einstein College of Medicine. Some of this work was published in a thesis submitted in partial fulfillment of the requirements for a Doctor of Philosophy conferred by the Graduate Program in Biomedical Sciences of the Albert Einstein College of Medicine (MMC). We also acknowledge the support of the Einstein-Montefiore Center for AIDS Research, funded by P30AI051519. None of the funders had any role in the design, analysis or interpretation of data; in the writing of the manuscript or in the decision to submit the manuscript for publication. T. gondii genomic and/or cDNA sequence data were accessed via http://ToxoDB.org. We would like to acknowledge the preliminary work of Pedro Pagan and Levy Vargas to test the feasibility of GSEA, and Omar Harb and Jeroen Saeij for providing gene lists.

\section{Author details}

${ }^{1}$ Departments of Medicine, Microbiology \& Immunology and Pathology, Albert Einstein College of Medicine, 1300 Morris Park Avenue, 10461 Bronx, NY, USA. ${ }^{2}$ Department of Biological Sciences, Hunter College of the City of University of New York, New York 10065, NY, USA. ${ }^{3}$ Departments of Molecular Medicine and Global Health, University of South Florida, Tampa 33612, FL, USA.

Received: 18 April 2014 Accepted: 17 June 2014

Published: 24 June 2014

\section{References}

1. Knoll L, Tomita T, Weiss LM: Bradyzoite Development. In Toxoplasma gondii The Model Apicomplexan: Perspectives and Methods. 2nd edition. Edited by Weiss LM, Kim K. New York: Academic Press; 2014:521-551.

2. Subramanian A, Tamayo P, Mootha VK, Mukherjee S, Ebert BL, Gillette MA, Paulovich A, Pomeroy SL, Golub TR, Lander ES, Mesirov JP: Gene set enrichment analysis: a knowledge-based approach for interpreting genome-wide expression profiles. Proc Natl Acad Sci U S A 2005, 102:15545-15550.

3. Ashburner M, Ball CA, Blake JA, Botstein D, Butler H, Cherry JM, Davis AP, Dolinski K, Dwight SS, Eppig JT, Harris MA, Hill DP, Issel-Tarver L, Kasarskis A, Lewis S, Matese JC, Richardson JE, Ringwald M, Rubin GM, Sherlock G: Gene ontology: tool for the unification of biology. The gene ontology consortium. Nat Genet 2000, 25:25-29.

4. Cherry JM, Hong EL, Amundsen C, Balakrishnan R, Binkley G, Chan ET, Christie KR, Costanzo MC, Dwight SS, Engel SR, Fisk DG, Hirschman JE, Hitz BC, Karra K, Krieger CJ, Miyasato SR, Nash RS, Park J, Skrzypek MS, Simison M, Weng S, Wong ED: Saccharomyces genome database: the genomics resource of budding yeast. Nucleic Acids Res 2012, 40:D700-D705.

5. Gajria B, Bahl A, Brestelli J, Dommer J, Fischer S, Gao X, Heiges M, lodice J, Kissinger JC, Mackey AJ, Pinney DF, Roos DS, Stoeckert CJ, Wang H, Brunk BP: ToxoDB: an integrated Toxoplasma gondii database resource. Nucleic Acids Res 2008, 36:D553-D556.

6. Kanehisa M, Goto S, Sato Y, Furumichi M, Tanabe M: KEGG for integration and interpretation of large-scale molecular data sets. Nucleic Acids Res 2012, 40:D109-D114.

7. Seeber F, Feagin JE, Parsons M: The Apicoplast and Mitochondrion of Toxoplasma Gondii. In Toxoplasma gondii The Model Apicomplexan: Perspectives and Methods. 2nd edition. Edited by Weiss LM, Kim K. New York: Academic Press; 2014: 298-351.

8. Lebrun M, Carruthers VB, Cesbron-Delauw M-F: Toxoplasma Secretory Proteins and Their Roles in Cell Invasion and Intracellular Survival. In Toxoplasma Gondii Model Apicomplexan Perspect. Methods. 2nd edition. Edited by Weiss LM, Kim K. New York: Academic Press; 2014: 390-455.

9. Buchholz KR, Fritz HM, Chen X, Durbin-Johnson B, Rocke DM, Ferguson DJ, Conrad PA, Boothroyd JC: Identification of tissue cyst wall components by transcriptome analysis of in vivo and in vitro Toxoplasma gondii bradyzoites. Eukaryot Cell 2011, 10:1637-1647.

10. Fritz HM, Buchholz KR, Chen X, Durbin-Johnson B, Rocke DM, Conrad PA, Boothroyd JC: Transcriptomic analysis of toxoplasma development reveals many novel functions and structures specific to sporozoites and oocysts. PLoS One 2012, 7:e29998.

11. Lescault PJ, Thompson AB, Patil V, Lirussi D, Burton A, Margarit J, Bond J, Matrajt M: Genomic data reveal Toxoplasma gondii differentiation mutants are also impaired with respect to switching into a novel extracellular tachyzoite state. PLoS One 2010, 5:e14463.

12. Naguleswaran A, Elias EV, McClintick J, Edenberg HJ, Sullivan WJ Jr: Toxoplasma gondii lysine acetyltransferase GCN5-A functions in the cellular response to alkaline stress and expression of cyst genes. PLoS Pathog 2010, 6:e1001232.

13. Reich M, Liefeld T, Gould J, Lerner J, Tamayo P, Mesirov JP: GenePattern 2.0. Nat Genet 2006, 38:500-501.

14. Behnke MS, Wootton JC, Lehmann MM, Radke JB, Lucas O, Nawas J, Sibley $L D$, White MW: Coordinated progression through two subtranscriptomes underlies the tachyzoite cycle of Toxoplasma gondii. PLoS One 2010, 5:e12354 
15. Gubbels MJ, White M, Szatanek T: The cell cycle and Toxoplasma gondii cell division: tightly knit or loosely stitched? Int J Parasitol 2008, 38:1343-1358.

16. Bahl A, Davis PH, Behnke M, Dzierszinski F, Jagalur M, Chen F, Shanmugam D, White MW, Kulp D, Roos DS: A novel multifunctional oligonucleotide microarray for Toxoplasma gondii. BMC Genomics 2010, 11:603.

17. Bohne W, Roos DS: Stage-specific expression of a selectable marker in Toxoplasma gondii permits selective inhibition of either tachyzoites or bradyzoites. Mol Biochem Parasitol 1997, 88:115-126.

18. Radke JR, Guerini MN, Jerome M, White MW: A change in the premitotic period of the cell cycle is associated with bradyzoite differentiation in Toxoplasma gondii. Mol Biochem Parasitol 2003, 131:119-127.

19. Yahiaoui B, Dzierszinski F, Bernigaud A, Slomianny C, Camus D, Tomavo S: Isolation and characterization of a subtractive library enriched for developmentally regulated transcripts expressed during encystation of Toxoplasma gondii. Mol Biochem Parasitol 1999, 99:223-235.

20. Zhang YW, Halonen SK, Ma YF, Wittner M, Weiss LM: Initial characterization of CST1, a Toxoplasma gondii cyst wall glycoprotein. Infect Immun 2001, 69:501-507.

21. Tomita T, Bzik DJ, Ma YF, Fox BA, Markillie LM, Taylor RC, Kim K, Weiss LM: The Toxoplasma gondii cyst wall protein CST1 is critical for cyst wall integrity and promotes bradyzoite persistence. PLoS Pathog 2013, 9:e1003823.

22. Behnke MS, Radke JB, Smith AT, Sullivan WJ Jr, White MW: The transcription of bradyzoite genes in Toxoplasma gondii is controlled by autonomous promoter elements. Mol Microbiol 2008, 68:1502-1518.

23. Le Roch KG, Zhou Y, Blair PL, Grainger M, Moch JK, Haynes JD, De La Vega P, Holder AA, Batalov S, Carucci DJ, Winzeler EA: Discovery of gene function by expression profiling of the malaria parasite life cycle. Science 2003, 301:1503-1508.

24. Bozdech Z, Llinás M, Pulliam BL, Wong ED, Zhu J, DeRisi JL: The transcriptome of the intraerythrocytic developmental cycle of Plasmodium falciparum. PLOS Biol 2003, 1:E5.

25. Pino P, Foth BJ, Kwok L-Y, Sheiner L, Schepers R, Soldati T, Soldati-Favre D: Dual targeting of antioxidant and metabolic enzymes to the mitochondrion and the apicoplast of Toxoplasma gondii. PLoS Pathog 2007, 3:e115.

26. Shanmugasundram A, Gonzalez-Galarza FF, Wastling JM, Vasieva O, Jones AR: Library of Apicomplexan Metabolic Pathways: a manually curated database for metabolic pathways of apicomplexan parasites. Nucleic Acids Res 2013, 41:D706-D713.

27. Tomavo S, Boothroyd JC: Interconnection between organellar functions, development and drug resistance in the protozoan parasite. Toxoplasma gondii. Int J Parasitol 1995, 25:1293-1299.

28. Narasimhan J, Joyce BR, Naguleswaran A, Smith AT, Livingston MR, Dixon SE, Coppens I, Wek RC, Sullivan WJ Jr: Translation regulation by eukaryotic initiation factor-2 kinases in the development of latent cysts in Toxoplasma gondii. J Biol Chem 2008, 283:16591-16601.

29. Joyce BR, Konrad C, Wek RC, Sullivan WJ Jr: Translation control is critical during acute and chronic stages of toxoplasmosis infection. Expert Rev Anti Infect Ther 2011, 9:1-3.

30. Gaji RY, Behnke MS, Lehmann MM, White MW, Carruthers VB: Cell cycle-dependent, intercellular transmission of Toxoplasma gondii is accompanied by marked changes in parasite gene expression. Mol Microbiol 2011, 79:192-204.

31. Balaji $S$, Babu MM, Iyer LM, Aravind L: Discovery of the principal specific transcription factors of Apicomplexa and their implication for the evolution of the AP2-integrase DNA binding domains. Nucleic Acids Res 2005, 33:3994-4006.

32. Altschul SF, Wootton JC, Zaslavsky E, Yu Y-K: The construction and use of log-odds substitution scores for multiple sequence alignment. PLoS Comput Biol 2010, 6:e1000852.

33. Campbell TL, De Silva EK, Olszewski KL, Elemento O, Llinás M: Identification and genome-wide prediction of DNA binding specificities for the ApiAP2 family of regulators from the malaria parasite. PLoS Pathog 2010, 6:e1001165.

doi:10.1186/1471-2164-15-515

Cite this article as: Croken et al:: Gene Set Enrichment Analysis (GSEA)

of Toxoplasma gondii expression datasets links cell cycle progression and the bradyzoite developmental program. BMC Genomics 2014 15:515.

\section{Submit your next manuscript to BioMed Central and take full advantage of:}

- Convenient online submission

- Thorough peer review

- No space constraints or color figure charges

- Immediate publication on acceptance

- Inclusion in PubMed, CAS, Scopus and Google Scholar

- Research which is freely available for redistribution 